\title{
Autophagy in breast cancer metastatic dormancy: tumor suppressing or tumor promoting functions?
}

\author{
Alyssa La Belle Flynn', William P. Schiemann² \\ 'Department of Pharmacology, Case Western Reserve University, Cleveland, OH 44106, USA. \\ ${ }^{2}$ Case Comprehensive Cancer Center, Case Western Reserve University, Cleveland, OH 44106, USA.
}

Correspondence to: Dr. William P. Schiemann, Case Comprehensive Cancer Center, Case Western Reserve University, Wolstein Research Building, Room 2131, 2103 Cornell Road, Cleveland, OH 44106, USA. E-mail: william.schiemann@case.edu

How to cite this article: Flynn ALB, Schiemann WP. Autophagy in breast cancer metastatic dormancy: tumor suppressing or tumor promoting functions? J Cancer Metastasis Treat 2019;5:43. http://dx.doi.org/10.20517/2394-4722.2019.13

Received: 5 Feb 2019 First Decision: 4 Mar 2019 Revised: 27 Mar 2019 Accepted: 16 Apr 2019 Published: 14 May 2019

Science Editor: Chun Hei Antonio Cheung Copy Editor: Cai-Hong Wang Production Editor: Huan-Liang Wu

\begin{abstract}
Breast cancer is the second leading cause of cancer-associated death in women in the United States, with more than $90 \%$ of those deaths attributed to metastasis. Breast cancer metastasis is incurable and possesses few treatment options and a poor overall prognosis due in part to confounding metastatic attributes, particularly the acquisition of dormancy-associated phenotypes. Dormant disseminated tumor cells can persist for years-to-decades before recurring as highly aggressive, secondary lesions. Dormancy-associated phenotypes are exhibited by breast cancer stem cells (BCSCs), which undergo tumor initiation and unlimited self-renewal. In addition to their specialized abilities to circumvent chemotherapeutic insults, BCSCs also upregulate autophagy during metastatic dormancy as a means to survive in nutrient poor conditions and environmental stress. As such, therapeutic targeting of autophagy is actively being pursued as an attractive strategy to alleviate metastatic disease and the recurrence of dormant BCSCs. Here we review the molecular and cellular features of autophagy, as well as its paradoxical role in both suppressing and promoting mammary tumor development and metastatic progression. Finally, we highlight the clinical challenges associated with therapeutic targeting of autophagy in metastatic breast cancers.
\end{abstract}

Keywords: Autophagy, breast cancer, cancer stem cells, metastatic dormancy, metastatic relapse

\section{INTRODUCTION}

Breast cancer is the second deadliest malignancy in women, accounting for nearly 41,000 deaths in the United States in $2018^{[1]}$. More than $90 \%$ of the deaths attributed to breast cancer are caused by metastasis, a disease state associated with poor prognosis and little-to-no effective treatment options ${ }^{[2]}$. Indeed, while initial treatment of breast cancers can be effective and achieve remission, an estimated $30 \%$ of lymph

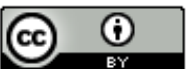

(C) The Author(s) 2019. Open Access This article is licensed under a Creative Commons Attribution 4.0 International License (https://creativecommons.org/licenses/by/4.0/), which permits unrestricted use, sharing, adaptation, distribution and reproduction in any medium or format, for any purpose, even commercially, as long as you give appropriate credit to the original author(s) and the source, provide a link to the Creative Commons license, and indicate if changes were made.

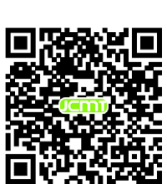


node-negative and 70\% of lymph node-positive breast cancer patients will eventually relapse 5-20 years following initial diagnosis ${ }^{[3,4]}$. The period of time between clinical remission and relapse can be attributed to dormancy, a process whereby disseminated tumor cells (DTCs) enter a non-proliferative state coupled with the activation of cellular stress programs ${ }^{[5]}$. Even in the earliest stages of mammary tumor development, breast cancer cells are actively shed from the growing tumor and traverse the metastatic cascade before colonizing distant metastatic sites ${ }^{[6,7]}$. These solitary micrometastases can persist in distant organs for years or even decades before emerging as recurrent metastatic tumors. Indeed, experimental evidence and in silico modeling indicate that dormant DTCs exist in a quiescent state as opposed to one that reflects a balance between cell proliferation and apoptosis ${ }^{[8-12]}$. Dormant cells upregulate pro-survival factors and are inherently chemoresistant given their non-proliferative state. As such, treatment with currently available therapeutics does little to limit the population of dormant cells in breast cancer patients. In fact, $\sim 62 \%$ of breast cancerassociated deaths occur 5 years following diagnosis ${ }^{[13]}$. As such, the clinical detection and treatment of these recurrent metastases remains challenging due to: (1) difficulties in detecting growing lesions years or decades following remission; (2) limited treatment options that are effective against metastatic disease ${ }^{[14,15]}$. Despite the fact that systemic relapse following a period metastatic dormancy remains a large unmet clinical burden, the precise mechanism(s) that enable dormant metastatic lesions to reactivate proliferative programs and recur remains incomplete ${ }^{[3]}$. Here we highlight the importance of breast cancer stem cells (BCSCs) and their reliance upon autophagy to govern the activation and eventual emergence from metastatic dormancy, as well as clinical implications of targeting autophagy therapeutically as a means to alleviate metastatic disease.

\section{BCSCS AND METASTATIC DORMANCY: A ROUTE TO EVADE DETECTION AND THERAPEUTIC ELIMINATION}

Recent evidence suggests that DTCs endowed with the ability to survive metastatic dormancy and initiate recurrent metastatic lesions are $\mathrm{BCSCs}^{[16-18]}$, which undergo unlimited self-renewal and contribute to tumor initiation ${ }^{[19]}$. Likewise, genomic analyses of primary and relapsed metastatic breast cancers reveal numerous common driver mutations shared between primary and metastatic tumor lesions in a given patient. As such, these common mutational landscapes implicate the presence of a common malignant cell of origin and support the notion that disseminated BCSCs initiate recurrent metastatic lesions years or decades following clinical remission ${ }^{[20-23]}$. This process reflects the ability of BCSCs to adopt dormancy-associated phenotypes through several malleable events, including modulation of E-cadherin and lncRNA expression ${ }^{[24,25]}$. Equally important facets of metastatic relapse are the capacity of BCSCs to evade immune surveillance and resist therapeutic interventions aimed at eradicating residual disease. Amongst the pro-survival strategies activated by BCSCs are: (1) upregulated expression of ATP-binding cassette transporters that mediate cellular efflux of chemotherapeutic agents ${ }^{[26-28]} ;$ (2) increased production of Interleukin-4 (IL-4) to suppress apoptosis $^{[29]}$; (3) enhanced generation of reactive oxygen species in response to radiation ${ }^{[30]}$; (4) elevated activation of autophagy ${ }^{[16-18,31]}$ [Figure 1]. As such, dormant BCSCs are inherently resistant to traditional chemotherapeutic agents and radiation that target rapidly dividing tumor cells. In the succeeding sections, we highlight the role of autophagy in regulating mammary tumorigenesis and dormancy-associated phenotypes during metastatic progression and relapse.

\section{CONTEXT-DEPENDENT ROLE OF AUTOPHAGY IN TUMOR PROGRESSION}

Macroautophagy (hereafter referred to as autophagy) is a highly conserved process that maintains cellular homeostasis through the lysosomal degradation of proteins and organelles, a phenomenon that is tightly controlled by autophagy-related genes (ATGs) ${ }^{[32]}$. The autophagosome cargo protein, p62/sequestosome 1 (SQSTM1), binds to degradation targets and facilitates selective autophagy ${ }^{[33]}$. Indeed, during the activation of autophagy, ATGs mediate the recycling of p62/SQSTM1-tagged cargo through the formation of doublemembrane vesicles, termed autophagosomes, which fuse with lysosomes to form autophagolysosomes. Lysosomal fusion facilitates the degradation of nonfunctional cellular components and also functions 


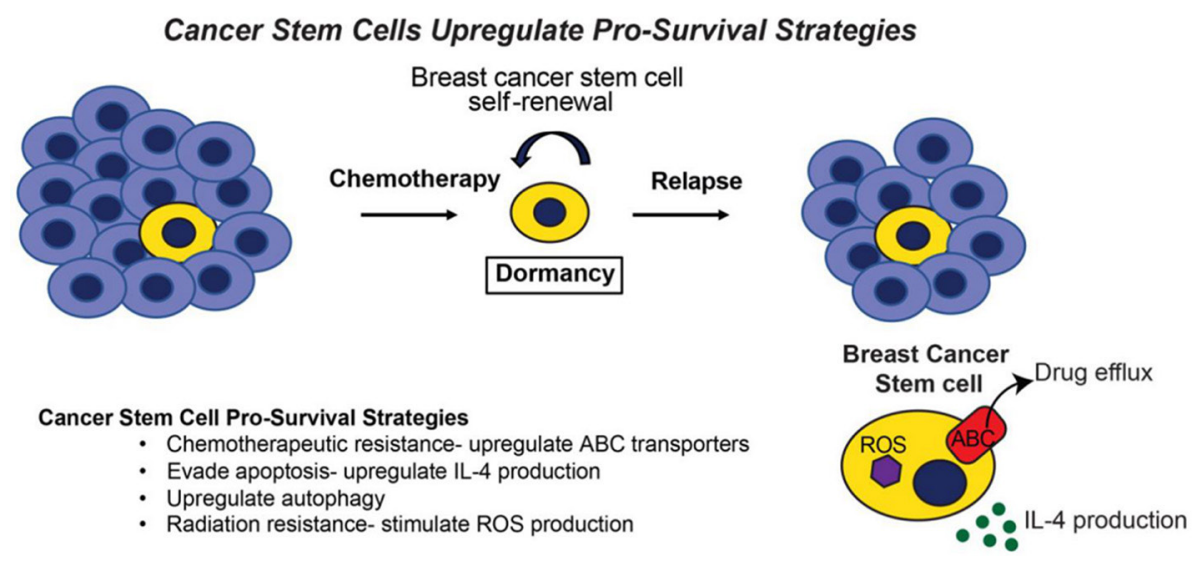

Figure 1. Cancer stem cells upregulate pro-survival strategies. Early in mammary tumor development, breast cancer cells are shed and disseminated from the growing lesion, ultimately colonizing distant metastatic sites before clinical detection of a primary breast tumor. Upon breast cancer diagnosis, neoadjuvant chemotherapy in conjunction with surgical resection, or more traditionally, surgery followed by adjuvant chemotherapy are both effective in eliminating the bulk the primary tumor cells. In contrast to bulk tumor cells, breast cancer stem cells manage to survive chemotherapeutic treatment by upregulating a number of pro-survival strategies, thereby contributing to metastatic relapse following a period of remission and dormancy. In doing so, cancer stem cells can (1) upregulate ABC transporter expression, which evades the cytotoxic activities of chemotherapies; (2) enhance IL-4 production, which inhibits apoptosis; (3) activate autophagy; (4) induce ROS production, which confers resistance to radiation. In addition, breast cancer stem cells also evade apoptosis by lying dormant for years or even decades, a pathophysiological state that further protects these cells from the cytotoxic activities of chemotherapy and radiation, and from the apoptotic activities engendered by metabolic, hypoxic, and environmental stressors

to meet the energy demands of a cell in periods of environmental stress ${ }^{[32,34]}$ [Figure 2]. Recent basic and clinical research findings have highlighted the context-dependent role of autophagy in regulating tumorigenesis. Indeed, in the earliest stages of tumor growth and development, autophagy functions as a tumor suppressor, thereby limiting tumor growth. However, once primary tumors or their metastases are established, autophagy can promote tumorigenesis by subverting stress responses, and consequently, facilitating tumor cell survival and disease progression ${ }^{[35]}$ [Figure 3]. At present, a thorough understanding of the molecular mechanisms that enable autophagy to both suppresses or promote mammary tumorigenesis is lacking, as are cell- and context-specific signals that underlie the paradoxical functions of autophagy in breast cancers. Future studies need to address these important questions as a means to uncover novel therapeutic strategies aimed at modulating autophagy in patients with metastatic breast cancer.

\section{AUTOPHAGY AND TUMOR SUPPRESSION}

Anecdotal evidence indicates that autophagy can act as a barrier to prevent tumor initiation in a number of solid tumors, including those of the breast. For instance, autophagy is readily induced by the tumor suppressors PTEN and p53, while their inactivation in developing neoplasms inhibits autophagy, as does oncogenic activation of PI3K/AKT and $\mathrm{BCL} 22^{[36]}$. Likewise, monoallelic deletion of the autophagy regulator, beclin-1, is observed in $40 \%-75 \%$ of breast and ovarian human tumors ${ }^{[37]}$, suggesting that autophagy functions to suppress tumor initiation. Accordingly, genetic inactivation of beclin-1 in mice predisposes their development of a variety of tumors, findings consistent with the notion that autophagy regulates cellular homeostasis and prevents tumor initiation ${ }^{[37-39]}$. Furthermore, activation of the transcription factor NRF2 elicits deregulation of autophagy due in part to aberrant accumulation of p62/SQSTM1 that can promote tumor formation ${ }^{[40-42]}$. Indeed, under tonic conditions, NRF2 interacts with Keap1, which targets NRF2 for ubiquitin-mediated degradation. The interaction between Keap1 and NRF2 can be prevented by the accumulation of p62/SQSTM1, thereby: (1) inhibiting the activation of autophagy; (2) stabilizing NRF2 expression, leading to its transcriptional activation ${ }^{[40-43]}$. Finally, emerging evidence suggests a role for autophagy in maintaining genomic integrity, as metabolic stress induced by loss of autophagy can promote DNA damage and chromosomal instability ${ }^{[44]}$. Indeed, when confronted with DNA damage, autophagy- 


\section{Autophagy: A Pro-Survival Strategy Employed by Breast Cancer Stem Cells}

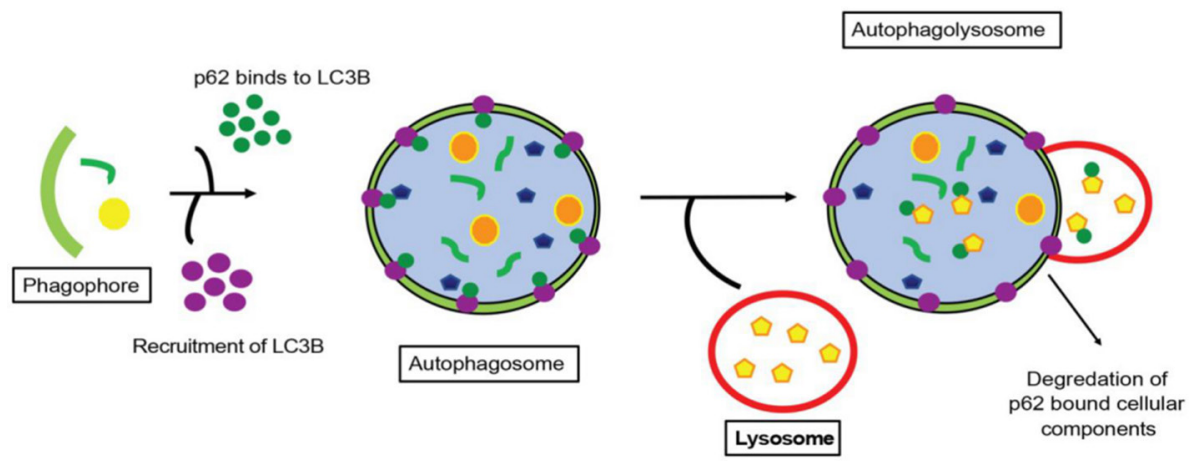

Figure 2. Autophagy: a pro-survival strategy employed by breast cancer stem cells. One of the pro-survival strategies employed by breast cancer stem cells during their acquisition of dormant states is autophagy, which facilitates the recycling of damaged or unnecessary organelles and/or proteins as a means to provide energy during periods of metabolic stress. Upon initiation of autophagy, the phagophore encircles those cellular contents targeted for autophagic degradation. LC3 is recruited to the phagophore and subsequently binds to the cargo adaptor protein, p62/SQSTM1. Upon doing so, a double membrane structure called the autophagosome forms and encircles cellular candidates for autophagic degradation. Subsequently, the autophagosome binds to the highly acidic lysosome to form the autophagolysosome, wherein p62/SQSTM1 bound cellular contents are degraded

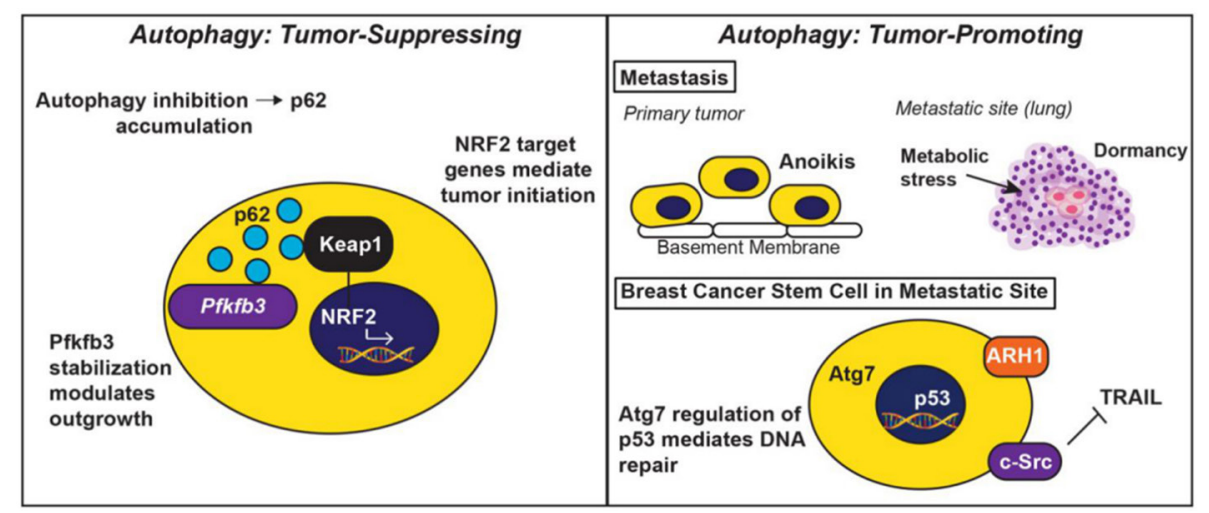

Figure 3. The tumor-suppressing and tumor-promoting activities elicited by autophagy. Autophagy functions to suppress tumor initiation (left panel), as well as to promote tumor development and progression (right panel). In early stages of tumor formation or during periods of metastatic dormancy, autophagy is tumor suppressive. Upon autophagy inhibition, p62/SQSTM1 accumulates and stabilizes Pfkfb3, leading dormant breast cancer stem cells to initiate metastatic relapse. Additionally, p62/SQSTM1 also inhibits the interaction between Keap1 and NRF2, thereby preventing NRF2-mediated expression of genes operant in tumor initiation (left panel). In stark contrast, autophagy provides established tumors with pro-survival phenotypes, including protection from anoikis and intrinsic cellular stressors encountered during metastatic dormancy. Likewise, autophagy protects breast cancer stem cells by ensuring for their resistance to the apoptotic stimuli housed within the metastatic microenvironment (e.g., Src-mediated TRAIL resistance), and to chemotherapeutic insults (e.g., Atg7-mediated p53 regulation of DNA repair). Finally, dormant cells can upregulate ARH1 to induce autophagy and promote the activation of pro-survival signaling systems that ensure for their survival

deficient cells exhibit diminished homologous recombination (HR) repair of damaged DNA that arises due to proteasomal degradation of checkpoint kinase $1(\mathrm{Chk} 1)^{[45,46]}$. While non-homologous end joining (NHEJ) appears to be largely unaffected by autophagy inhibition, the diminished HR proficiency in these cells can render them more sensitive to DNA damage, especially if NHEJ is subsequently impaired ${ }^{[45,46]}$. Collectively, these findings identify important mechanisms whereby autophagy functions to suppress malignant transformation and tumor development.

\section{AUTOPHAGY AND TUMOR PROMOTION}

In contrast to its tumor suppressing functions, autophagy can also serve as a tumor promoting process, particularly by: (1) enhancing the ability of DTCs to traverse the metastatic cascade; (2) inhibiting 
immunosurveillance by tumor infiltrating immune cells. During metastasis, cells shed from the primary tumor must invade through the extracellular matrix, intravasate into blood vessels, survive the turbid flow of the vasculature, extravasate, and finally colonize a distant metastatic site ${ }^{[47]}$. Not surprisingly, cells traversing the metastatic cascade experience a variety of cellular stressors and vastly different tissue microenvironments, including changes in the (1) composition of the extracellular matrix composition; (2) availability of nutrients due to alterations in vascular and lymphatic networks; (3) biomechanical properties of metastatic sites; (4) tumor immunosurveillance programs ${ }^{[48-50]}$. Importantly, autophagy activation protects DTCs during periods of metabolic stress encountered by anoikis and entry into foreign microenvironments ${ }^{[51-53]}$, and by bouts of dormancy at distant metastatic sites ${ }^{[54,55]}$.

\section{AUTOPHAGY INHIBITS TUMOR IMMUNOSURVEILLANCE}

Tumor immunosurveillance is a critical physiological process that inhibits the development and progression of mammary tumors. Accumulating data in the literature indicate that tumor immunosurveillance programs are an all-encompassing system that involves not only the adaptive immune system and cytotoxic effector pathways, but also the release of a complex set of cytokines and chemokines that coalesce to prevent tumor development ${ }^{[50]}$. Although the molecular mechanisms used by tumor cells to escape immunosurveillance are varied, recent evidence has implicated a role for autophagy in mediating this phenomenon. Indeed, autophagy activation has been shown to inhibit immune cell killing of tumor cells as a means to promote escape from immunosurveillance and DTC outgrowth ${ }^{[50]}$. In doing so, autophagy activation can target the activation of Signal Transducer and Activator of Transcription 3 (STAT3), a gene commonly dysregulated in breast cancer that also plays a prominent role in regulating the immune system ${ }^{[56]}$. For instance, the activation of autophagy can induce the phosphorylation and stimulation of STAT3 in tumors, an event that initiates cellular cross-talk between tumor and immune cells that ultimately suppresses Cytotoxic T Lymphocyte (CTL)-mediated lysis of tumor cells ${ }^{[56,57]}$. Likewise, autophagy can inhibit natural killer cell (NK)-mediated tumor cell killing by degrading granzyme B, a serum protease that is released by NK cells during NK-mediated cell killing ${ }^{[58,59]}$. Finally, breast cancer development and metastatic progression is critically dependent upon Epithelial-Mesenchymal Transition (EMT) programs ${ }^{[60]}$. Interestingly, autophagy is activated as carcinoma cells traverse the EMT program, with the resulting post-EMT mesenchymallike cells exhibiting elevated levels of autophagy relative to their pre-EMT epithelial-like counterparts. Importantly, EMT-mediated activation of autophagy inhibits CTL-mediated antitumor immunosurveillance in a beclin-1-dependent manner ${ }^{[50,61-63]}$. Collectively, these studies highlight the tumor intrinsic and extrinsic functions of autophagy, with the latter function, fulfilling an essential role in governing the fidelity of tumor immunosurveillance mechanisms.

\section{DORMANT BCSCS AND AUTOPHAGY ACTIVATION}

BCSCs can lie dormant for decades before recurring as metastatic lesions in breast cancer patients. During this time, disseminated BCSCs must survive nonpermissive tumor environments, while simultaneously maintaining their viability and the capacity for tumor initiation ${ }^{[5,64]}$. Emerging evidence implicates autophagy as an essential feature in maintaining the phenotypes associated with BCSCs, particularly their resistance to chemotherapies and hypoxic microenvironments ${ }^{[16,18,54,55,65-67]}$. The mechanisms whereby autophagy promotes BCSC survival at metastatic sites are varied and include the ability to confer resistance to apoptotic stimuli (e.g., Src-mediated TRAIL resistance in bone metastases ${ }^{[68]}$ ), to chemotherapeutic insults (e.g., DNA repair via Atg7 and p53 by $A \operatorname{tg} 7^{[69]}$ ), and to cellular stressors ${ }^{[70]}$. Similarly, aberrant expression of ARHI (aplasia Ras homolog member 1) can elicit autophagy activation and modulate the survival of dormant cells in preclinical models of ovarian cancer, further implicating autophagy as an essential mediator of dormant cell survival $^{[71]}$. Finally, tumor cells that possess defects in autophagy readily accumulate p62/SQSTM1, an event that alters p62/SQSTM1 function and contributes to tumorigenesis ${ }^{[17]}$. The aforementioned studies highlight the oncogenic and pro-survival activities of autophagy that contribute to tumor progression, as well as the 
acquisition and eventual emergence from metastatic dormancy. In the succeeding sections, we discuss the implications of targeting the dichotomous roles of autophagy in clinical settings.

\section{CLINICAL TARGETING AND MODULATION OF AUTOPHAGY}

Numerous clinical trials have aimed with varying degrees of success to inhibit or stimulate autophagy as a potential cancer therapeutic ${ }^{[35]}$. Despite significant investments in preclinical and clinical investigations, no FDA-approved drugs designed to modulate autophagy have been approved for the treatment of primary or metastatic breast cancers. This clinical deficit reflects the challenges associated with the dichotomous roles played by autophagy during mammary tumor development and metastatic progression, and with the inability of science and medicine to fully appreciate the downstream consequences of autophagy modulation in metastatic disease settings.

\section{INHIBITORS OF AUTOPHAGY}

At present, nearly 32 human clinical trials have been undertaken to assess the efficacy of autophagy modulating agents [Table 1], either administered alone or in combination with standard-of-care chemotherapeutics (www.clinicaltrials.gov). Pharmacological inhibition of autophagy in clinical settings is primarily accomplished using chloroquine, or a closely related molecule, hydroxychloroquine. Chloroquine functions to block autophagosome-lysosome fusion by preventing the acidification of the lysosome, thus inhibiting autophagy ${ }^{[72,73]}$. While the vast majority of studies include either chloroquine or hydroxychloroquine in combination with standard-of-care regimens, one recent study utilized a novel proteasome inhibitor, MLN9708, as a means to assess the impact of autophagy in conferring breast cancer resistance to the cytotoxic activities of doxorubicin ${ }^{[7]}$. Interestingly, administration of MLN9708 to breast cancer cells resulted in autophagy activation in a manner paralleling previous connections between proteasomal inhibitor and autophagy ${ }^{[75,76]}$. Moreover, MLN9708 enhanced the sensitivity of breast cancer cells to doxorubicin in a manner that was inversely correlated with the extent of autophagy activation ${ }^{[74]}$. As such, future studies need to assess the effectiveness of combining proteasomal and autophagy inhibitors with cytotoxic chemotherapies (e.g., doxorubicin).

Additional translational insights into how autophagy inhibition impacts cancer cell survival has been accomplished using a combination of pharmacologic (e.g., choloroquine and/or hydroxychloroquine) and genetic (e.g., knockdown of autophagy associated genes) approaches. In general, these studies support the concept that inactivation of autophagy limits the development and spread of human cancers. Interestingly, recent evidence indicates that the molecular mechanisms underlying the cytotoxic activities of chloroquine and hydroxychloroquine are distinct from those employed to inhibit autophagy. Indeed, induction of lysosomal membrane permeabilization was insufficient to elicit apoptosis in cells treated with chloroquine. Rather, the cytotoxic activities of chloroquine were found to manifest subsequent to mitochondrial membrane permeabilization ${ }^{[7]}$, and to reduced expression and activity of JAK3 and DNMT1 ${ }^{[78]}$. Precisely how these alternative targets and activities attributed to chloroquine contribute to its clinical successes and failures remains an important line of research in the field of autophagy modulation.

\section{STIMULATORS OF AUTOPHAGY}

In light of the dichotomous activities autophagy plays during tumorigenesis, clinical investigation has also evaluated the impact of stimulating autophagy as a means to limit the growth and spread of cancers. Indeed, mTOR (mammalian target of rapamycin) is the primary pathway targeted pharmacologically as a means to induce autophagy in human breast cancers. For instance, several studies have investigated the importance of $\operatorname{rapamycin}^{[79]}$, Everolimus ${ }^{[80,81]}$, and Temsirolimus ${ }^{[80,82]}$ as potential inducers of autophagy in clinical settings. In general, autophagy activation elicited in response to mTORC1 inactivation is a byproduct of the intended 
Table 1. Cancer clinical trials evaluating autophagy modulation

\begin{tabular}{|c|c|c|c|}
\hline Clincial trial title & Status & Conditions & Interventions \\
\hline Autophagy Bladder Cancer & Not yet recruiting & Bladder Cancer & \\
\hline $\begin{array}{l}\text { Sorafenib Induced Autophagy Using } \\
\text { Hydroxychloroquine in Hepatocellular Cancer }\end{array}$ & Recruiting & Hepatocellular Cancer & $\begin{array}{l}\text { Drug: Sorafenib (SOR)|Drug: } \\
\text { Hydroxychloroquine (HCQ) }\end{array}$ \\
\hline $\begin{array}{l}\text { A Phase I/II/Pharmacodynamic Study of } \\
\text { Hydroxychloroquine in Combination With } \\
\text { Gemcitabine/Abraxane to Inhibit Autophagy }\end{array}$ & $\begin{array}{l}\text { Active, not } \\
\text { recruiting }\end{array}$ & $\begin{array}{l}\text { Advanced Adenocarcinoma|Metastatic } \\
\text { Adenocarcinoma }\end{array}$ & $\begin{array}{l}\text { Drug: Hydroxychloroquine } \\
\text { (HCQ)|Drug: Gemcitabine }\end{array}$ \\
\hline
\end{tabular}

\section{in Pancreatic Cancer}

Hydroxychloroquine, Palbociclib, and Recruiting Letrozole Before Surgery in Treating

Participants With Estrogen Receptor Positive,

HER2 Negative Breast Cancer
Imaging Tumor Hypoxia With 18F-EF5 PET in Active, not Recurrent or Metastatic Clear Cell Ovarian recruiting Cancer

Enzalutamide and Metformin Hydrochloride Active, not in Treating Patients With Hormone-Resistant recruiting Prostate Cancer

Vorinostat Plus Hydroxychloroquine Versus Recruiting Regorafenib in Colorectal Cancer

\section{Sirolimus or Vorinostat and} Hydroxychloroquine in Advanced Cancer

MLN9708 and Vorinostat in Patients With Advanced p53 Mutant Malignancies

Akt Inhibitor MK2206 and

Hydroxychloroquine ir Advanced Solid Tumors, Melanoma, Prostate or Kidney Cancer

Active, not recruiting

Active, not recruiting Active, not
Hydroxychloroquine + Vorinostat in Advanced Solid Tumors

Novel Molecular Targets for Ductal Carcinoma In Situ (DCIS)

Observational Study of Biomarker During Liver Surgery

Pantoprazole and Docetaxel for Men With Metastatic Castration-Resistant Prostate Cancer

Active, not recruiting

Active, not recruiting Recruiting

Active, not recruiting
Anatomic Stage I Breast Cancer AJCC v8|Anatomic Stage IA Breast Cancer AJCC v8|Anatomic Stage IB Breast Cancer AJCC v8|Anatomic Stage II Breast Cancer AJCC v8|Anatomic Stage IIA Breast Cancer AJCC v8|Anatomic Stage IIB Breast Cancer AJCC v8|Anatomic Stage III Breast Cancer AJCC v8|Anatomic Stage IIIA Breast Cancer AJCC v8|Anatomic Stage IIIB Breast Cancer AJCC v8|Anatomic Stage IIIC Breast Cancer AJCC v8|Anatomic Stage IV Breast Cancer AJCC v8|Estrogen Receptor Positive|HER2/Neu Negative|MKI67 Positive|Postmenopaus al|Prognostic Stage I Breast Cancer AJCC v8|Prognostic Stage IA Breast Cancer AJCC v8|Prognostic Stage IB Breast Cancer AJCC v8|Prognostic Stage IIA Breast Cancer AJCC v8|Prognostic Stage IIB Breast Cancer AJCC v8|Prognostic Stage III Breast Cancer AJCC v8|Prognostic Stage IIIA Breast Cancer AJCC v8|Prognostic Stage IIIB Breast Cancer AJCC v8|Prognostic Stage IIIC Breast Cancer AJCC v8|Prognostic Stage IV Breast Cancer AJCC v8 Ovarian Cancer|Ovarian Neoplasms

Drug: 18F-EF5 PET/CT scan|Procedure: Optional biopsy

Prostate Cancer

Drug: Enzalutamide|Drug: Metformin Hydrochloride

Advanced Cancers
Drug: Hydroxychloroquine|Drug: Letrozole|Drug: Palbociclib
Colorectal Cancer

Advanced Cancers

Adult Solid Neoplasm|HormoneResistant Prostate Carcinoma|Recurrent Melanoma|Recurrent Prostate Carcinoma|Recurrent Renal Cell Carcinoma|Stage IIIA Cutaneous Melanoma AJCC v7|Stage IIIB Cutaneous Melanoma AJCC v7|Stage IIIC Cutaneous Melanoma AJCC v7|Stage IV Cutaneous Melanoma AJCC v6 and v7|Stage IV Prostate Cancer AJCC v7|Stage IV Renal Cell Cancer AJCC v7

Drug: Vorinostat|Drug:

Hydroxychloroquine|Drug: Regorafenib

Drug: Hydroxychloroquine|Drug: Sirolimus|Drug: Vorinostat

Drug: MLN9708|Drug: Vorinostat

Drug: Akt Inhibitor MK2206|Drug:

Hydroxychloroquine|Other:

Laboratory Biomarker Analysis|Other:

Pharmacological Study Malignant Solid Tumour

Breast Cancer

Drug: Hydroxychloroquine|Drug:

Vorinostat

Other: Biomarkers

Liver Cancer|Hepatobiliary Tract Adenomas and Carcinomas

Procedure: Hepatocellular carcinom

Drug: Pantoprazole
Prostate Cancer 
A Randomized, 2×2 Factorial Design Biomarker Prevention Trial of Low-dose

Recruiting

Tertiary Prevention in Colon Cancer

Aspirin and Metformin in Stage I-III

Colorectal Cancer Patients.

Randomized Phase II Trial of Pre-Operative Active, not

Gemcitabine and Nab Paclitacel With or With recruiting

Out Hydroxychloroquine

The Treatment of Advanced Lung Cancer

With Dribbles Antigen by Targeting

Activation of Tcells

Pre-Operative Trial (PGHA vs. PGH) for

Not yet recruiting Carcinoma, Non-Small-Cell Lung

Recruiting Pancreatic Cancer Resectable

Resectable Pancreatic Cancer

Sunitinib Malate and Hydroxychloroquine in Treating Patients With Advanced Solid

Active, not

Adult Solid Neoplasm

Tumors That Have Not Responded to

Chemotherapy

The Role of Fibroblast Activation in Uterine Not yet recruiting Uterine Fibroid

Fibroid

International Cooperative Phase III Trial of the Recruiting HIT-HGG Study Group (HIT-HGG-2013)

\author{
Short Course Radiation Therapy With \\ Proton or Photon Beam Capecitabine and \\ Hydroxychloroquine for Resectable Pancreatic \\ Cancer \\ Androgen Deprivation Therapy Muscle \\ Protein Metabolism and Blood Glucose \\ Autophagy Inhibition to Augment mTOR \\ Inhibition: A Phase I/II Trial of RAD001 \\ and Hydroxychloroquine in Patients With \\ Previously Treated Renal Cell Carcinoma \\ Dabrafenib/Trametinib/Hydroxychloroquine Recruiting \\ for Advanced Pretreated BRAF V600 Mutant \\ Melanoma \\ The Addition of Chloroquine to \\ Chemoradiation for Glioblastoma, \\ The Addition of Chloroquine to \\ Chemoradiation for Glioblastoma \\ Gemcitabine, Docetaxel, and \\ Hydroxychloroquine in Treating Participants \\ With Recurrent or Refractory Osteosarcoma \\ The BAMM Trial: BRAF, Autophagy and \\ MEK Inhibition in Metastatic Melanoma: \\ A Phase I/2 Trial of Dabrafenib, Trametinib \\ and Hydroxychloroquine in Patients With \\ Advanced BRAF Mutant Melanoma \\ Characterization of the Mechanisms of \\ Resistance to Azacitidine
}

Active, not
recruiting

Recruiting

Active, not

recruiting

Glioblastoma WHO Grade IV|Diffuse Midline Glioma Histone 3 K27M WHO Grade IV|Anaplastic Astrocytoma WHO Grade III|Diffuse Intrinsic Pontine Glioma|Gliomatosis Cerebri

Pancreatic Cancer

Prostate Cancer|Resistance Exercise|Androgen Deprivation Therapy

Metastatic Clear Cell Renal Cell

Carcinoma

Melanoma

Not yet recruiting Glioblastoma|Astrocytoma, Grade IV

Recruiting Glioblastoma Multiforme

Recruiting

Recurrent Osteosarcoma|Refractory Osteosarcoma

Recruiting

Advanced BRAF Mutant Melanoma

TN-TC11G (THC+CBD) Combination With Temozolomide and Radiotherapy in Patients With Newly-diagnosed Glioblastoma

Hepatocellular Carcinoma in Patients With Recruiting

a Cirrhosis Due to an Alcoholic or a Non

Alcoholic Fatty Liver Disease

List of clinical trials (www.clinicaltrials.gov) that are currently active, recruiting, or not yet recruiting patients for clinical trials to study how autophagy modulation, primarily through chloroquine or hydroxychloroquine treatment, influences tumor growth and progression

drug target, thereby producing synergistic cell killing in the form of autosis (i.e., autophagic cell $\mathrm{death}^{[83]}$ ). Along these lines, several clinical trials associated with mTOR modulation have sought to overcome endocrine resistance associated with hormone receptor positive breast cancer treatments. Unfortunately, single agent modification of autophagy by administration of mTOR pathway inhibitors has proven to be
Drug: Aspirin (ASA) + Metformin Placebos

Drug: gemcitabine|Drug: abraxane|Drug: hydroxychloroquine

Biological: Dribble vaccine

Drug: Gemcitabine, NabPaclitaxel, hydroxychloroquine and Avelumab|Drug: Gemcitabine, NabPaclitaxel, and hydroxychloroquine

Drug: Hydroxychloroquine|Other: Laboratory Biomarker Analysis|Other: Pharmacological Study|Drug: Sunitinib Malate

Genetic: Measurement of protein expression in tissue and /or blood samples.

Drug: Temozolomide + Valproic

Acid|Drug: Temozolomide +

Chloroquine

Drug: Capecitabine|Drug:

Hydroxychloroquine|Radiation:

Proton or Photon Radiation Therapy

Drug: Zoladex

Drug: Hydroxychloroquine|Drug: RAD001

Drug: Dabrafenib|Drug:

Trametinib|Drug: Hydroxychloroquine

Drug: Chloroquine

Drug: Chloroquine|Radiation: Radiotherapy|Drug: Temozolomide

Drug: Docetaxel|Drug:

Gemcitabine|Drug:

Hydroxychloroquine

Drug: Trametinib 2 mg daily|Drug: hydroxychloroquine (HCQ)

Drug: TN-TC11G|Drug: Temozolomide Oral Product|Radiation: Radiotherapy

Other: blood collection
Myelodysplastic Syndromes or Acute
Myeloid Leukemia With Multilineage Dysplasia

Hepatocellular Carcinoma 
highly ineffective at restoring endocrine sensitivity to estrogen receptor-positive breast cancers. Likewise, combining autophagy modulators with anti-estrogens has also failed to significantly improve the clinical course of these patients, with severe toxicities being associated with Everolimus ${ }^{[80]}$. Thus, similar to the strategy of autophagy inhibition, the clinical utility of autophagy activation to eliminate metastatic breast cancers awaits additional mechanistic and translational investigation.

\section{CONCLUSION}

Metastatic dormancy is mediated by BCSCs and responsible for the majority of breast cancer-associated deaths. An inherent property of BCSCs reflects their ability to activate a variety of pro-survival strategies to circumvent metabolic stress within the metastatic niche, and to overcome therapeutic insults mediated by chemotherapies and radiation. The activation of autophagy has proven to be a critical component of the pro-survival strategies employed by BCSCs, especially when confronted with nutrient deprivation, with inhospitable tissue microenvironments, with cytotoxic agents, and with dormancy-associated phenotypes. Indeed, preclinical evidence implicates important roles for autophagy modulation in the treatment of breast cancer. However, the paradoxical functions of autophagy to both suppress and promote tumorigenesis has clearly hampered the development and implementation of effective autophagy modulators for the treatment of metastatic breast cancer. Accordingly, several important avenues of basic and clinical investigation need to be achieved in order to generate effective autophagic agents. First, studies need to determine the extent to which chemotherapeutic drugs rely upon autophagy modulation when inducing their cytotoxic activities in target cells. Indeed, these so-called "off-target" effects on autophagy may underscore either directly or indirectly the extent to which a therapeutic regimen is effective, or alternatively, is rendered insensitive. Second, additional efforts need to be directed at identifying improved autophagy modulating drugs, particularly those that are effective against metastatic disease. Third, enhancing our understanding of how the tumor microenvironment impacts the targeting of autophagy-directed drugs is also warranted ${ }^{[84-86]}$. Finally, efforts directed at developing biomarkers capable of identifying patients most likely to benefit from autophagy modulation needs to be undertaken to minimize potential untoward side effects (e.g., disease progression, emergence from dormancy, and metastatic relapse) of this course of treatment. Ultimately, addressing these research avenues will provide new inroads for strategies aimed at targeting autophagy vulnerability in BCSCs, and consequently, at eliminating metastatic relapse.

\section{DECLARATIONS}

\section{Acknowledgments}

Members of the Schiemann Laboratory are thanked for critical comments and reading of the manuscript.

\section{Author's contributions}

Conception and study design: Flynn ALB, Schiemann WP

Drafted and revised the manuscript: Flynn ALB, Schiemann WP

\section{Availability of data and materials}

Not applicable.

\section{Financial support and sponsorship}

Research support was provided in part by the National Institutes of Health (CA236273) to Schiemann WP, and (T32GM008803 and T32CA059366) to Flynn ALB. Additional support was graciously provided by the METAvivor Foundation to Schiemann WP, and by pilot funding from the Case Comprehensive Cancer Center's Research Innovation Fund, which is supported by the Case Council and Friends of the Case Comprehensive Cancer Center to Schiemann WP, and from the Case Clinical \& Translational Science Collaborative to Schiemann WP. 


\section{Conflicts of interest}

All authors declare that there are no conflicts of interest.

\section{Ethical approval and consent to participate}

Not applicable.

\section{Consent for publication}

Not applicable.

\section{Copyright}

(c) The Author(s) 2019.

\section{REFERENCES}

1. Siegel RL, Miller KD, Jemal A. Cancer statistics, 2018. CA Cancer J Clin 2018;68:7-30.

2. O'Shaughnessy J. Extending survival with chemotherapy in metastatic breast cancer. Oncologist 2005;10 Suppl 3:20-9.

3. Cardoso F, Harbeck N, Fallowfield L, Kyriakides S, Senkus E, et al. Locally recurrent or metastatic breast cancer: ESMO clinical practice guidelines for diagnosis, treatment and follow-up. Ann Oncol 2012;23 Suppl 7:vii11-9.

4. Foulkes WD, Smith IE, Reis-Filho JS. Triple-negative breast cancer. N Engl J Med 2010;363:1938-48.

5. Sosa MS, Bragado P, Aguirre-Ghiso JA. Mechanisms of disseminated cancer cell dormancy: an awakening field. Nat Rev Cancer 2014;14:611-22.

6. Yoshida BA, Sokoloff MM, Welch DR, Rinker-Schaeffer CW. Metastasis-suppressor genes: a review and perspective on an emerging field. J Natl Cancer Inst 2000;92:1717-30.

7. Cowin P, Welch DR. Breast cancer progression: controversies and consensus in the molecular mechanisms of metastasis and EMT. J Mammary Gland Biol Neoplasia 2007;12:99-102.

8. Taylor DP, Wells JZ, Savol A, Chennubhotla C, Wells A. Modeling boundary conditions for balanced proliferation in metastatic latency. Clin Cancer Res 2013;19:1063-70.

9. Wells A, Griffith L, Wells JZ, Taylor DP. The dormancy dilemma: quiescence versus balanced proliferation. Cancer Res 2013;73:3811-6.

10. Gao H, Chakraborty G, Lee-Lim AP, Mo Q, Decker M, et al. The BMP inhibitor Coco reactivates breast cancer cells at lung metastatic sites. Cell 2012;150:764-79.

11. Chao YL, Shepard CR, Wells A. Breast carcinoma cells re-express E-cadherin during mesenchymal to epithelial reverting transition. Mol Cancer 2010;9:179.

12. Marshall JC, Collins JW, Nakayama J, Horak CE, Liewehr DJ, et al. Effect of inhibition of the lysophosphatidic acid receptor 1 on metastasis and metastatic dormancy in breast cancer. J Natl Cancer Inst 2012;104:1306-19.

13. Klein CA. Framework models of tumor dormancy from patient-derived observations. Curr Opin Genet Dev 2011;21:42-9.

14. Demicheli R, Abbattista A, Miceli R, Valagussa P, Bonadonna G. Time distribution of the recurrence risk for breast cancer patients undergoing mastectomy: further support about the concept of tumor dormancy. Breast Cancer Res Treat 1996;41:177-85.

15. Hensel JA, Flaig TW, Theodorescu D. Clinical opportunities and challenges in targeting tumour dormancy. Nat Rev Clin Oncol 2013;10:41-51.

16. Carcereri de Prati A, Butturini E, Rigo A, Oppici E, Rossin M, et al. Metastatic Breast Cancer Cells Enter Into Dormant State and Express Cancer Stem Cells Phenotype Under Chronic Hypoxia. J Cell Biochem 2017;118:3237-48.

17. Mathew R, Karp CM, Beaudoin B, Vuong N, Chen G, et al. Autophagy suppresses tumorigenesis through elimination of p62. Cell 2009; 137:1062-75.

18. Mowers EE, Sharifi MN, Macleod KF. Autophagy in cancer metastasis. Oncogene 2017;36:1619-30.

19. Prager BC, Xie Q, Bao S, Rich JN. Cancer stem cells: the architects of the tumor ecosystem. Cell Stem Cell 2019;24:41-53.

20. Ding L, Ellis MJ, Li S, Larson DE, Chen K, et al. Genome remodelling in a basal-like breast cancer metastasis and xenograft. Nature 2010;464:999-1005.

21. Yates LR, Gerstung M, Knappskog S, Desmedt C, Gundem G, et al. Subclonal diversification of primary breast cancer revealed by multiregion sequencing. Nat Med 2015;21:751-9.

22. Shah SP, Morin RD, Khattra J, Prentice L, Pugh T, et al. Mutational evolution in a lobular breast tumour profiled at single nucleotide resolution. Nature 2009;461:809-13.

23. Savas P, Teo ZL, Lefevre C, Flensburg C, Caramia F, et al. The subclonal architecture of metastatic breast cancer: results from a prospective community-based rapid autopsy program "CASCADE". PLoS Med 2016;13:e1002204.

24. Wendt MK, Taylor MA, Schiemann BJ, Schiemann WP. Downregulation of epithelial cadherin is required to initiate metastatic outgrowth of breast cancer. Mol Bio cell 2011;22:2423-35.

25. Gooding AJ, Zhang B, Jahanbani FK, Gilmore HL, Chang JC, et al. The lncRNA BORG drives breast cancer metastasis and disease recurrence. Sci Rep 2017;7:12698.

26. Dean M. ABC transporters, drug resistance, and cancer stem cells. J Mammary Gland Biol Neoplasia 2009;14:3-9.

27. Donnenberg VS, Meyer EM, Donnenberg AD. Measurement of multiple drug resistance transporter activity in putative cancer stem/ progenitor cells. Methods Mol Biol 2009;568:261-79. 
28. Hawley TS, Riz I, Yang W, Wakabayashi Y, Depalma L, et al. Identification of an ABCB1 (P-glycoprotein)-positive carfilzomib-resistant myeloma subpopulation by the pluripotent stem cell fluorescent dye CDy1. Am J Hematol 2013;88:265-72.

29. Todaro M, Alea MP, Di Stefano AB, Cammareri P, Vermeulen L, et al. Colon cancer stem cells dictate tumor growth and resist cell death by production of interleukin-4. Cell Stem Cell 2007;1:389-402.

30. Diehn M, Cho RW, Lobo NA, Kalisky T, Dorie MJ, et al. Association of reactive oxygen species levels and radioresistance in cancer stem cells. Nature 2009;458:780-3.

31. Zou Z, Yuan Z, Zhang Q, Long Z, Chen J, et al. Aurora kinase A inhibition-induced autophagy triggers drug resistance in breast cancer cells. Autophagy 2012;8:1798-810.

32. White E, Mehnert JM, Chan CS. Autophagy, metabolism, and cancer. Clin Cancer Res 2015;21:5037-46.

33. Rogov V, Dotsch V, Johansen T, Kirkin V. Interactions between autophagy receptors and ubiquitin-like proteins form the molecular basis for selective autophagy. Mol Cell 2014;53:167-78.

34. Galluzzi L, Pietrocola F, Bravo-San Pedro JM, Amaravadi RK, Baehrecke EH, et al. Autophagy in malignant transformation and cancer progression. EMBO J 2015;34:856-80.

35. Thorburn A, Thamm DH, Gustafson DL. Autophagy and cancer therapy. Mol Pharmacol 2014;85:830-8.

36. Kimmelman AC. The dynamic nature of autophagy in cancer. Genes Dev 2011;25:1999-2010.

37. Liang XH, Jackson S, Seaman M, Brown K, Kempkes B, et al. Induction of autophagy and inhibition of tumorigenesis by beclin 1 . Nature 1999;402:672-6.

38. Yue Z, Jin S, Yang C, Levine AJ, Heintz N. Beclin 1, an autophagy gene essential for early embryonic development, is a haploinsufficient tumor suppressor. Proc Natl Acad Sci U S A 2003;100:15077-82.

39. Qu X, Yu J, Bhagat G, Furuya N, Hibshoosh H, et al. Promotion of tumorigenesis by heterozygous disruption of the beclin 1 autophagy gene. J Clin Invest 2003;112:1809-20.

40. Komatsu M, Kurokawa H, Waguri S, Taguchi K, Kobayashi A, et al. The selective autophagy substrate p62 activates the stress responsive transcription factor Nrf2 through inactivation of Keap1. Nat Cell Biol 2010;12:213-23.

41. Lau A, Wang XJ, Zhao F, Villeneuve NF, Wu T, et al. A noncanonical mechanism of Nrf2 activation by autophagy deficiency: direct interaction between Keap1 and p62. Mol Cell Biol 2010;30:3275-85.

42. Villeneuve NF, Lau A, Zhang DD. Regulation of the Nrf2-Keap1 antioxidant response by the ubiquitin proteasome system: an insight into cullin-ring ubiquitin ligases. Antioxid Redox Signal 2010;13:1699-712.

43. Hayes JD, McMahon M. NRF2 and KEAP1 mutations: permanent activation of an adaptive response in cancer. Trends Biochem Sci 2009;34:176-88.

44. Mathew R, Kongara S, Beaudoin B, Karp CM, Bray K, et al. Autophagy suppresses tumor progression by limiting chromosomal instability. Genes Dev 2007;21:1367-81.

45. Liu EY, Xu N, O'Prey J, Lao LY, Joshi S, et al. Loss of autophagy causes a synthetic lethal deficiency in DNA repair. Proc Natl Acad Sci U S A 2015;112:773-8.

46. Gillespie DA, Ryan KM. Autophagy is critically required for DNA repair by homologous recombination. Mol Cell Oncol 2016;3:e1030538.

47. Massague J, Obenauf AC. Metastatic colonization by circulating tumour cells. Nature 2016;529:298-306.

48. Wyckoff JB, Jones JG, Condeelis JS, Segall JE. A critical step in metastasis: in vivo analysis of intravasation at the primary tumor. Cancer Res 2000;60:2504-11.

49. Chambers AF, Groom AC, MacDonald IC. Dissemination and growth of cancer cells in metastatic sites. Nat Rev Cancer 2002;2:563-72.

50. Janji B, Viry E, Moussay E, Paggetti J, Arakelian T, et al. The multifaceted role of autophagy in tumor evasion from immune surveillance. Oncotarget 2016;7:17591-607.

51. Degenhardt K, Mathew R, Beaudoin B, Bray K, Anderson D, et al. Autophagy promotes tumor cell survival and restricts necrosis, inflammation, and tumorigenesis. Cancer Cell 2006;10:51-64.

52. Karantza-Wadsworth V, Patel S, Kravchuk O, Chen G, Mathew R, et al. Autophagy mitigates metabolic stress and genome damage in mammary tumorigenesis. Genes Dev 2007;21:1621-35.

53. Fung C, Lock R, Gao S, Salas E, Debnath J. Induction of autophagy during extracellular matrix detachment promotes cell survival. Mol Biol Cell 2008;19:797-806.

54. Cufi S, Vazquez-Martin A, Oliveras-Ferraros C, Martin-Castillo B, Vellon L et al. Autophagy positively regulates the CD44(+) CD24(-/ low) breast cancer stem-like phenotype. Cell Cycle 2011;10:3871-85.

55. Vera-Ramirez L, Vodnala SK, Nini R, Hunter KW, Green JE. Autophagy promotes the survival of dormant breast cancer cells and metastatic tumour recurrence. Nat Commun 2018;9:1944.

56. Yu H, Kortylewski M, Pardoll D. Crosstalk between cancer and immune cells: role of STAT3 in the tumour microenvironment. Nat Rev Immunol 2007;7:41-51.

57. Kortylewski M, Kujawski M, Wang T, Wei S, Zhang S et al. Inhibiting Stat3 signaling in the hematopoietic system elicits multicomponent antitumor immunity. Nat Med 2005;11:1314-21.

58. Baginska J, Viry E, Berchem G, Poli A, Noman MZ et al. Granzyme B degradation by autophagy decreases tumor cell susceptibility to natural killer-mediated lysis under hypoxia. Proc Natl Acad Sci U S A 2013;110:17450-5.

59. Viry E, Baginska J, Berchem G, Noman MZ, Medves S et al. Autophagic degradation of GZMB/granzyme B: a new mechanism of hypoxic tumor cell escape from natural killer cell-mediated lysis. Autophagy 2014;10:173-5.

60. Taylor MA, Parvani JG, Schiemann WP. The pathophysiology of epithelial-mesenchymal transition induced by transforming growth factor-beta in normal and malignant mammary epithelial cells. J Mammary Gland Biol Neoplasia 2010;15:169-90.

61. Ricciardi M, Zanotto M, Malpeli G, Bassi G, Perbellini O et al. Epithelial-to-mesenchymal transition (EMT) induced by inflammatory priming elicits mesenchymal stromal cell-like immune-modulatory properties in cancer cells. Br J Cancer 2015;112:1067-75. 
62. Akalay I, Janji B, Hasmim M, Noman MZ, Andre F et al. Epithelial-to-mesenchymal transition and autophagy induction in breast carcinoma promote escape from T-cell-mediated lysis. Cancer Res 2013;73:2418-27.

63. Akalay I, Janji B, Hasmim M, Noman MZ, Thiery JP et al. EMT impairs breast carcinoma cell susceptibility to CTL-mediated lysis through autophagy induction. Autophagy 2013;9:1104-6.

64. Aguirre-Ghiso JA. Models, mechanisms and clinical evidence for cancer dormancy. Nat Rev Cancer 2007;7:834-46.

65. Chaffer CL, San Juan BP, Lim E, Weinberg RA. EMT, cell plasticity and metastasis. Cancer Metastasis Rev 2016;35:645-54.

66. Semenza GL. Hypoxia-inducible factors in physiology and medicine. Cell 2012;148:399-408.

67. Whelan KA, Chandramouleeswaran PM, Tanaka K, Natsuizaka M, Guha M, et al. Autophagy supports generation of cells with high CD44 expression via modulation of oxidative stress and Parkin-mediated mitochondrial clearance. Oncogene 2017;36:4843-58.

68. Zhang XH, Wang Q, Gerald W, Hudis CA, Norton L, et al. Latent bone metastasis in breast cancer tied to Src-dependent survival signals. Cancer Cell 2009;16:67-78.

69. Lee IH, Kawai Y, Fergusson MM, Rovira, II, Bishop AJ, et al. Atg7 modulates p53 activity to regulate cell cycle and survival during metabolic stress. Science 2012;336:225-8.

70. Lum JJ, Bauer DE, Kong M, Harris MH, Li C, et al. Growth factor regulation of autophagy and cell survival in the absence of apoptosis. Cell 2005;120:237-48.

71. Lu Z, Luo RZ, Lu Y, Zhang X, Yu Q, et al. The tumor suppressor gene ARHI regulates autophagy and tumor dormancy in human ovarian cancer cells. J Clin Invest 2008;118:3917-29.

72. Kimura T, Takabatake Y, Takahashi A, Isaka Y. Chloroquine in cancer therapy: a double-edged sword of autophagy. Cancer Res 2013;73:3-7.

73. Maycotte P, Aryal S, Cummings CT, Thorburn J, Morgan MJ, et al. Chloroquine sensitizes breast cancer cells to chemotherapy independent of autophagy. Autophagy 2012;8:200-12.

74. Wang H, Yu Y, Jiang Z, Cao WM, Wang Z, et al. Next-generation proteasome inhibitor MLN9708 sensitizes breast cancer cells to doxorubicin-induced apoptosis. Sci Rep 2016;6:26456.

75. Gavilan E, Giraldez S, Sanchez-Aguayo I, Romero F, Ruano D, et al. Breast cancer cell line MCF7 escapes from G1/S arrest induced by proteasome inhibition through a GSK-3beta dependent mechanism. Sci Rep 2015;5:10027.

76. Milani M, Rzymski T, Mellor HR, Pike L, Bottini A, et al. The role of ATF4 stabilization and autophagy in resistance of breast cancer cells treated with Bortezomib. Cancer Res 2009;69:4415-23.

77. Boya P, Gonzalez-Polo RA, Poncet D, Andreau K, Vieira HL, et al. Mitochondrial membrane permeabilization is a critical step of lysosome-initiated apoptosis induced by hydroxychloroquine. Oncogene 2003;22:3927-36.

78. Choi DS, Blanco E, Kim YS, Rodriguez AA, Zhao H, et al. Chloroquine eliminates cancer stem cells through deregulation of Jak2 and DNMT1. Stem Cells 2014;32:2309-23.

79. Royce ME, Osman D. Everolimus in the Treatment of Metastatic Breast Cancer. Breast Cancer (Auckl) 2015;9:73-9.

80. Vinayak S, Carlson RW. mTOR inhibitors in the treatment of breast cancer. Oncology (Williston Park) 2013;27:38-44, 6, 8 passim.

81. Ellard SL, Clemons M, Gelmon KA, Norris B, Kennecke H, et al. Randomized phase II study comparing two schedules of everolimus in patients with recurrent/metastatic breast cancer: NCIC Clinical Trials Group IND.163. J Clin Oncol 2009;27:4536-41.

82. Fleming GF, Ma CX, Huo D, Sattar H, Tretiakova M, et al. Phase II trial of temsirolimus in patients with metastatic breast cancer. Breast Cancer Res Treat 2012;136:355-63.

83. Liu Y, Shoji-Kawata S, Sumpter RM, Jr., Wei Y, Ginet V, et al. Autosis is a Na+,K+-ATPase-regulated form of cell death triggered by autophagy-inducing peptides, starvation, and hypoxia-ischemia. Proc Natl Acad Sci U S A 2013;110:20364-71.

84. Pavlides S, Tsirigos A, Migneco G, Whitaker-Menezes D, Chiavarina B, et al. The autophagic tumor stroma model of cancer: Role of oxidative stress and ketone production in fueling tumor cell metabolism. Cell Cycle 2010;9:3485-505.

85. Martinez-Outschoorn UE, Trimmer C, Lin Z, Whitaker-Menezes D, Chiavarina B, et al. Autophagy in cancer associated fibroblasts promotes tumor cell survival: Role of hypoxia, HIF1 induction and NFkappaB activation in the tumor stromal microenvironment. Cell Cycle 2010;9:3515-33.

86. Sotgia F, Martinez-Outschoorn UE, Howell A, Pestell RG, Pavlides S, et al. Caveolin-1 and cancer metabolism in the tumor microenvironment: markers, models, and mechanisms. Annu Rev Pathol 2012;7:423-67. 